\title{
THE TIME COURSE OF SACCADIC EYE MOVEMENTS IN GOLDFISH
}

\author{
S. S. EASTER. JR. \\ Department of Zoology, University of Michigan, Ann Arbor, Michigan 48104, U.S.A.
}

(Received 29 May 1974)

\begin{abstract}
Spontaneous saccadic eye movements $\left(2-25^{\circ}\right)$ made by goldfish have an initial brief acceleration. followed by a more prolonged deceleration. and occasionally a terminal phase during which the velocity reverses sign following overshoot of the final position. Overshoots by the individual eyes are independent events. The duration and the maximum velocity increase monotonically with the size of the saccade. Goldfish saccades have greater durations and slightly smaller maximum velocities than mammalian saccades of similar size.
\end{abstract}

\section{INTRODUCTION}

Saccadic eye movements are the very rapid shifts of position which have been observed in a wide variety of animals with mobile eyes. vertebrates and invertebrates alike. Primate saccades have been studied most completely, and one feature which stands oüt is their very great speed. Westheimer (1954) showed that human saccades typically last tenths of seconds and may reach velocities of several hundred degrees per second. Robinson (1964) and Fuchs (1967) studied saccades made by humans and monkeys and confirmed and extended Westheimer's results. The great speed was all the more remarkable following Robinson's (1964) analysis of the mechanics of the eye-orbital tissues system. which he found to be heavily overdamped. The high rotational velocities could only be achieved if tension exerted by the agonistic muscle had an initial transient much larger than the final value needed to hold the eye at its new position. Robinson's (1964) isometric measurements of extraocular muscle tensions during saccades confirmed that such a sequence of forces did occur.

These two forces. early and late. must be matched quite accurately if the saccade is to be executed smoothly. If the transient is too great or lasts too long. the saccade will overshoot the final value; if it is too small or brief. the eye will undershoot and slowly approach the final value from the direction of origin. Both of these phenomena have been observed (Weber and Daroff. 1972). which suggests that the transient and sustained tensions are occasionally mismatched. (For a discussion of the implications of this observation. see Easter. 1973, and Dell'Osso, Daroff and Troost. 1973.)

The only other animal in which saccades have been quantitatively studied is the rabbit (Collewijn. 1970). In the work reported below I have investigated saccadic eye movements in goldfish. and have concentrated on the time course and, indirectly, the coordination of transient and maintained tensions.

\section{METHODS}

Goldfish (Carassius auratus) $15-25 \mathrm{~cm}$ long. were obtained commercially (Ozark Fisheries. Stoutland. Mo.) and kept singly and in pairs in 10-gal aquaria. During an experiment. they were clamped in a sponge-lined holder. immersed in water $\left(20-22^{\circ} \mathrm{C}\right.$. the same temperature as the holding tanks). and rigid stalks were attached by suction to their corneas. Restrained goldfish spontaneously make saccades (Johnstone and Mark, 1969) which are essentially horizontal (Easter, 1971). As the eyes moved, so did the stalks, and the orientations of these pointers in the horizontal plane were measured by a flying spot scanner, the use and calibration of which have been described previously (Easter, 1971, 1972). Figure I schematizes the records. The

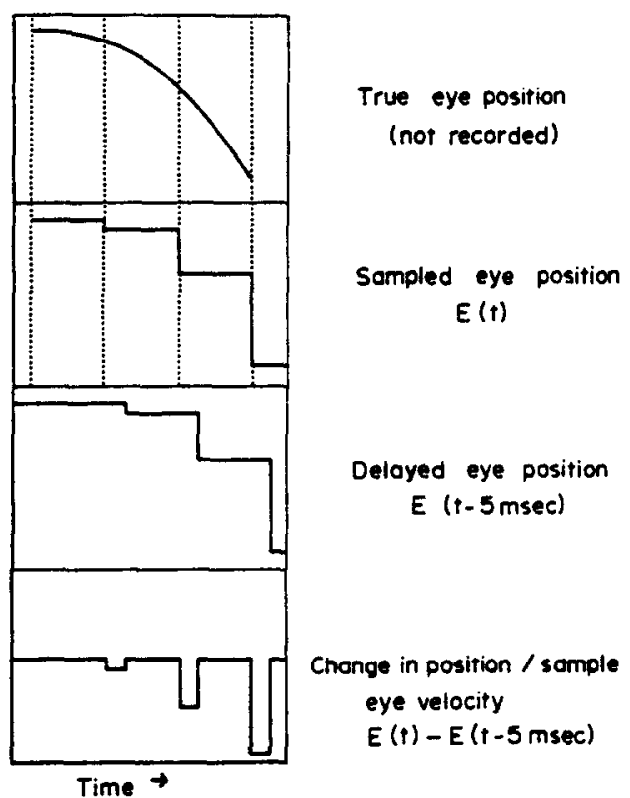

Fig. 1. This is a schematic representation of the electronic records of eye position and velocity. 
true eye position (top trace) was measured optically 60 times sec. and appeared as a voltage. $E(t)$ at the output of a sample and hold unit (second trace). This voltage is linearly related to the angular orientation of the eye in the horizontal plane. This same voltage, delayed by $5 \mathrm{msec}$, and called $E(t-5)$. appeared at the output of a second sample and hold unit (third trace). and was continuously subtracted electronically from $E(t)$. During most of the $16.7-\mathrm{msec} \mathrm{sam}$ pling interval. the difference was zero. but during the $5 \mathrm{msec}$ before the third trace has assumed the value of the second. they differed. and this difference in voltage (fourth trace) gives the velocity, the number of degrees moved in the previous $16.7 \mathrm{msec}$. Since the data were often recorded on a paper recorder, with a high frequency response of only (11) $\mathrm{H}$ / and this 5-msec pulse was too bricf to be recorded accurately. it was sampled by a third sample and hold unit and its output appeared on the paper record.

\section{Defintions and concentions}

The following definitions were used:

$T_{f}$. The durilian of the phase of acceleration. meatured by counting the number of velocity samples clearly distinguishable from the base line. beginning with the first and ending with the point of maximum deflection. This number is then multiplied by the sampling interval $(1 / 60 \mathrm{sec})$. In Fig. 2(a). lower trace, $T$, was $2,60 \mathrm{sec}$, or $33.3 \mathrm{msec}$.

$T_{2}$. The duration of the subsequent deceleration, obtained again from the velocity trace. and measured by counting the number of samples from the end of $T_{1}$ until the points cross. or become indistinguishable from. the base line. In Fig. 2(a). lower trace. $T_{2}$ is $6 / 60 \mathrm{sec}$ or $100 \mathrm{msec}$.

$T_{3}$. The duration of the entire saccade, equal to the sum of $\left(T_{1}+T_{2}\right)$ plus any additional time due to overshoot. In Fig. 2(a). lower trace. $T_{3}$ was $133.3 \mathrm{msec}$. Figure $2(\mathrm{c})$ shows a saccade which overshot the final value, yielding $T_{3}$ $(18.3 \mathrm{msec})$ greater than $\left(T_{1}+T_{2}\right)(116.7 \mathrm{msec})$.

$l_{m}$. The maximum velocity, that of the point deviating maximally from the baseline. In Fig. 2(a). lower trace. it is the second point above the base line. and corresponds to an angular velocity of $184^{\mathrm{J}} / \mathrm{sec}$. obtained by multiplying the voltage by the calibration factor, to get degrees. and this number by 60 . the sampling rate.

$W$. The magnitude. measured by subtracting the initial position from the position at $T_{3}$. In Fig. 2(a), upper trace, 11 was 9 ?.

By convention. eye movements which are counterclockwise when viewed from above are positive and appear directed upward on the records; clockwise movements are the

(c)

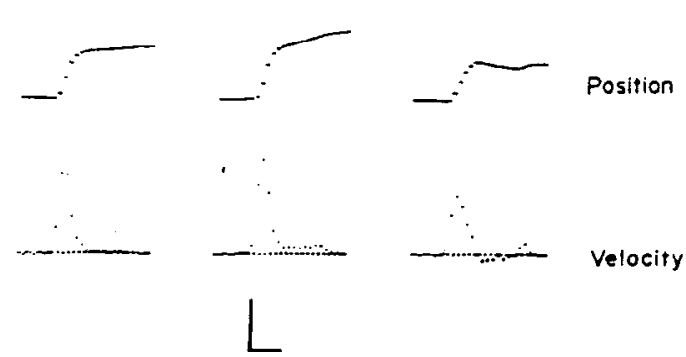

Fig. 2. These are oscillographs of individual saccades. all by the same fish. recorded as indicated in Fig. 1. Calibration: horizontal. $100 \mathrm{msec}$ : vertical, $10^{\circ}$ (upper records) $120^{\circ} / \mathrm{sec}$ (lower records) opposite. Thus when both eyes shift to the left, the left eye moving temporad. the right. nasad, a positive saccade has occurred

\section{RESLLTS}

\section{Effects of the stalks}

Before determining the time course. it was necessary to evaluate the effects of the stalks on $t_{m}$ and the $T$ 's. This was done by using stalks of several lengths on the same eye. and noting the time courses of the saccades.

The measurements went as follows. With the fish restrained. a stalk was attached to one eye. and the next 50 saccades (position and velocity records) were recorded on the two channel paper recorder. Then the stalk was replaced by another. of different length. and another 50 saccades were recorded. This was repeated until six sets of 50 saccades were obtained. The order of stalk lengths was: $0.7,1 \cdot 2,1 \cdot 7$. $2 \cdot 2.2 .7$ and $0.7 \mathrm{~cm}$. This required about $45 \mathrm{~min}$ to complete. after which the fish was returned to the holding tank.

Each saccade was scored for the five variables defined in the preceding section. Then the data within each of the six sets of 50 were pooled in multiple degree-wide bins of $|M|$. (bin width $=5.75^{\circ}$ in the example below) and the mean values of $T_{1}, T_{3}, T_{3}$ and $t_{m}$ were computed for each bin. The saccades made with larger stalks were compared with those made with the standard $(0.7 \mathrm{~cm}$. the shortest $)$ by computing ratios of the mean values of corresponding bins. For instance, during the first run. with $0.7 \mathrm{~cm}$ stalk, counterclockwise saccades ranging from 5.75 to $11.5^{\circ}$ had a mean $\left|r_{m}\right|$ of $117^{-} \mathrm{sec}$. When the stalk measuring $2.7 \mathrm{~cm}$ in length was on the eye, this same range of saccades reached a mean $\left|v_{m}\right|$ of only $57 \% \mathrm{sec}$. The ratio. $57 / 117$, was averaged with similarly computed ratios for saccades of all orher sizes made with these two stalk lengths in place. The final number. a mean
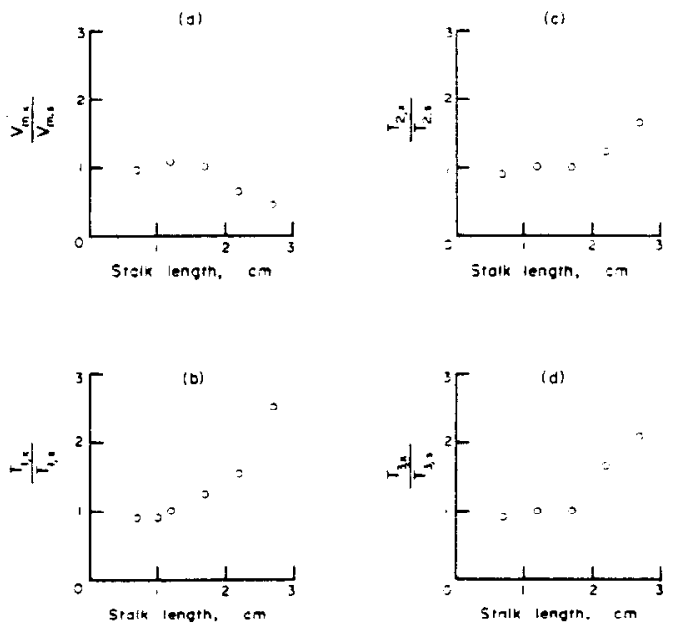

Fig. 3. These four graphs $(a-d)$ illustrate the effects of stalk length on the variables, $v_{m} T_{1}, T_{2}$ and $T_{3}$. which are defined in the text. The abscissa gives the length of the experimental stalk. The ordinate gives the ratio of the value obtained with the experimental stalk (subscript, $x$ ) over the value obtained with the standard stalk $(0.7 \mathrm{~cm}$, subscript. s). Stalks longer than $1.2 \mathrm{~cm}$ slowed (a) and prolonged (b-d) the saccades. 
value of ratios of $v_{\text {m }}$ corrected for $|M|$. indicates the effect of the longer stalks. These mean ratios are plotted in Fig. 3(a) where the abscissa gives the stalk length, and the ordinate. the ratio just described. Note that the left-most point. which givis the ratio $\left(r_{m}\right.$ of the last run $/ r_{m}$ of the first run), both of which were obtained with the same stalk is about unity. Therefore, the eye muscles had not fatigued during the experiment.

In Figs. $3(b)-(d)$ the relative durations of $T_{1}, T_{2}$ and $T_{3}$ are given again vs stalk length.

In all four graphs, the points for $0.7,1.2$ and $1.7 \mathrm{~cm}$ lengths are all about unity, indicating that the time course and maximum velocity of saccades were not afiected by stalks of these lengths. However, when the two longer stalks were used. duration increased and $\left|v_{m}\right|$ decreased. The increased duration stemmed principally from prolongation of the acceleratory phase [Fig. 3(b)] and addition of a third. often oscillatory phase. Both of these changes are consistent with the principal effect of the stalks being addition of sig. nificant moment of inertia. Robinson (1964) has shown that the moment of inertia of the orbital contents of primate eyes is negligible. It seems reasonable to assume that the same is true of the goldfish eye, in view of the structural similarities of the two.

The important point is that the data from the shorter stalks all lie on the horizontal corresponding to unity ratio, which suggests that the time course and velocity of saccades measured under these conditions are as they would be with no stalks. All the data described below were obtained on animals of about the same size as those used to generate Fig. 3. Stalks lengths were $1 \cdot 0-1 \cdot 2 \mathrm{~cm}$.

\section{Time course}

Examination of Fig. 2 reveals most of the qualitative features of saccades. The initial acceleratory phase rose quite abruptly from the noisy baseline. seldom lasted longer than $50 \mathrm{msec}$, and was followed by a more prolonged deceleration. This latter usually approached the baseline asymptotically, as seen in Fig. 2(a), but infrequently the velocity overshot or undershot. Overshoots resulted in oscillations, as illustrated in Fig. 2(c). Undershoots resulted in a terminal period of constant velocity, as shown in Fig. 2(b), but they were difficult to identify with any degree of assurance. In the example of Fig. 2(b) it is quite clear, but briefer terminal periods can not be so surely distinguished from the more common asymptotic approach. However, the overshoots were quite clear, and their occurrence was investigated in three fish to determine if they were systematically related to any other variable.

Each fish was restrained as usual, and both eyes monitored. Binocular saccades were recorded on a four-channel storage oscilloscope and immediately scored for $T_{1}, T_{2}, T_{3}$ and $M . M$ was scored to the nearest $\mathrm{mm}$ (i.e. $3.0^{\circ}$ of eye movement) on the oscilloscope scritn. T's were scored to the nearest $1 / 60$ sec. Fifty binocular saccades were recorded from each fish.

Of the 300 saccades examined, only 43 overshoots were seen, but 29 or these were made by the same fish. The other two made 11 and 3, respectively. This confirmed earlier impressions that the tendency to overshoot varied considerably from fish to fish. Although individual fish often showed a tendency to overshoot with one eye or in one direction. there was no systematic tendency for overshoots to be associated preferentially with one eye or with one direction.

The binocular independence of overshoots was investigated next. If an overshoot in one eye were independent of the occurence of an overshoot in the other, then the probability of simultaneous overshoots in both eyes should be predictable from the product of the individual probabilities of either. The animal which made 29 overshoots made 17 with the left eye and 12 with the right. The predicted number of simultaneous overshoots is:

$$
\frac{17}{50} \times \frac{12}{50} \times 50=4.15 .
$$

In fact. five were observed. A similar analysis for the other two fish yielded:

$$
\frac{1}{50} \times \frac{2}{50} \times 50=0.04
$$

and:

$$
\frac{1}{50} \times \frac{10}{50} \times 50=0.20 .
$$

The observed values were 0 and 1, respectively. These results support the hypothesis that overshoots were independent events; that is. a mismatched sequence of tensions in one eye was not generally accompanied by a mismatch in the other. This suggests that the motor programs of the two eyes are either independently generated. or at least independently susceptible to modification.

These same data werc analysed to reveal the dependence of $T_{1}, T_{2}$ and $T_{3}$ on $M$. There were quantitative differcnas between individualfish. but all werc alike in that larger saccades lasted longer, due almost entirely to increases in $T$. while $T_{1}$ was nearly constant. These observations are summarized in Fig. 4, data from the animal with the greatest range of $M$. The ordinate gives the mean value appropriate to all saccades of magnitude given by the abscissa. Saccades made by left and right eyes, in either direction, were pooled to obtain each mean. The nearly horizontal regression hotween $T_{1}$ and $M$ is quite different from the steep slope of the squares, which plot $T_{2}$ on $|M|$. Finally, the filled circles show that $T_{3}$, the duration of the entire saccade, increased also with $|M|$.

The dependence of $v_{m}$ on $M$ was assessed in a very similar way. In this case three (different) fish were used. one eye was monitored. and each saccade was scored for $v_{m}$ and $M$, the latter to $2 \cdot 0^{\circ}$ accuracy. Quantitative differences between individual fish were not so great as with the $T$ s, so the numbers from all three were pooled. Figure 5 shows the data arranged in $4^{\circ}$-wide bins of $|M|$, with the symbols representing the means. The $\left|v_{m}\right|$ increased monotonically with $|M|$ and reached values of several hundred degrees/sec. The values obtained by sampling at $60 \mathrm{~Hz}$ surely represent an underestimate of 


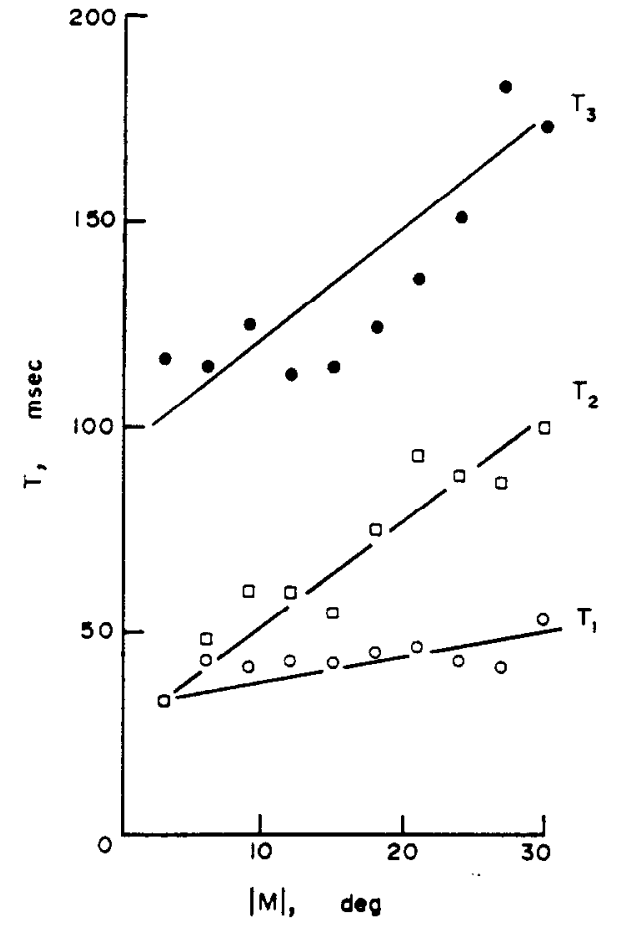

Fig. 4. This shows the dependence of $T_{1} . T_{2}$ and $T_{3}$ on $M$. for one fish. The lines are the least squares linear regressions.

the true $v_{m}$ as is obvious when one considers the velocity trace in Fig. 2(a), for example. The recorded $v_{m}$ was that of the second sample, $184^{\circ} / \mathrm{sec}$, but the most plausible continuous curve drawn through all the points would reach its peak some time between the second and third samples, at a value above the velocities recorded at those two times.

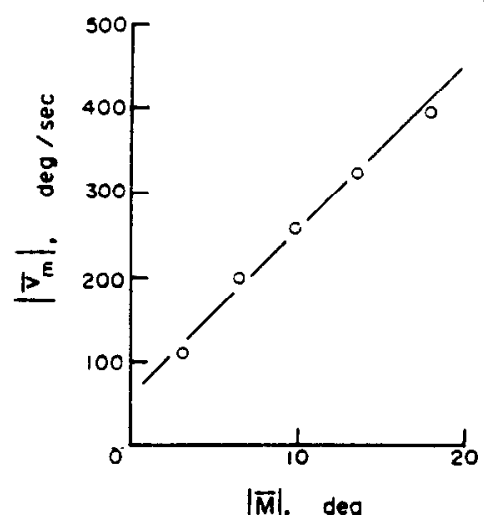

Fig. 5. This shows the dependence of $v_{m}$ on $|M|$ data pooled from three fish. The line is the least squares linear regression: $v_{m}=67+21|M|$ degrees $/$ sec.

\section{Disclission}

The measurements of the time course of saccades may be compared with the data from mammals. First. the $v_{m}$ 's are hundreds of degrees/sec, about the same as those reported for primates (Westheimer, 1954; Robinson. 1964: Fuchs. 1967) and rabbits (Collewijn. 1970). The profile of velocity vs time seems to be quite different in the fish. however, as the records of Robinson (1964, Fig. 1) and Fuchs (1967, Fig. 4) show that primate velocity was essentially constant for most of the saccade. The fish, on the other hand, never maintained a constant vilocity for long - the cyes were sither accelerating toward or decelerating from. the maximum value. It is unlikely that this difference is due to the presence of the measuring stalk on the fish's eye, since the main effect of the stalk was to increase the moment of inertia. which would oppose accelerations.

Another difference between primate and goldfish was the relation of $r_{m}$ and $M$. Becker and Fuchs (1969) found that $\left|v_{m}\right|$ increased roughly linearly as $|M|$ increased from 10 to $60^{\circ}$. but the increase only amounted to about $2^{\circ} / \mathrm{sec}$ per degree. In the goldfish. the slope was about tenfold greater (Fig. 5), but this difference may be due in part to the different ranges being compared. The data of Collewijn (1970) from rabbits more closely resemble the fish than the primate.

Finally, the duration of saccades was quite dependent on their magnitude, as in primates (Robinson, 1964). A useful summary relation is the least squares linear regression for data pooled from all three fish:

$$
T_{3}=67+2 \cdot 8|\mathrm{M}| \mathrm{msec} \text {. }
$$

(As was mentioned earlier, individual fish varied widely. For instance, the uppermost line in Fig. 4 intersects the ordinate at $95 \mathrm{msec}$ rather than 67.) This expression should be compared with Robinson's result for humans (Robinson, 1964; Alpern, 1969):

$$
T_{3}=21+2 \cdot 2 \mathrm{M} \text { msec. }
$$

and Fuchs' (1967) result for monkeys (estimated from Fig. 5 of that paper):

$$
T_{3}=18+1.1 \mathrm{Mmsec} .
$$

The goldfish saccades lasted longer, presumably because the high velocity, which fish and primates achieved about equally quickly, was not maintained by the fish.

Both Robinson (1964) and Fuchs (1967) noted differences in the durations of temporad and nasad saccades. No systematic differences were noticed in the fish, but this should not be taken to mean that there were none. In fact, the temporal resolution of the $60 \mathrm{~Hz}$ sampling rate would not detect differences as small as those noted in primates.

\section{SUMMARY}

(1) Spontaneous horizontal saccadic eye movements, $2-25^{\circ}$ in amplitude, made by restrained gold- 
fish, were measured. There were two and sometimes three phases.

(2) During the first phase the eye accelerated to its maximum velocity. The duration of this phase was roughly constant. between 33 and $50 \mathrm{msec}$ for saccades of all sizes.

(3) During the second phase, the eye decelerated back to zero velocity, and occasionally overshot the final position. The duration of this phase was usually greater than the first, and depended strongly on the magnitude of the saccade.

(4) During the (infrequent) third phase, the velocity reversed sign one or more times before the eye reached its final position.

(5) The duration and the maximum velocity were positively correlated with the magnitude of the saccade, as in mammals. Velocities reached several hundred degrees/sec, comparable with mammals. The durations were substantially longer than mammalian saccades of comparable size.

Acknowledgements-This work was supported by research grant EY-00168 from the National Eye Instituti of the United States Public Health Service. I thank Mr. M. MacLeod for typing and Mr. D. Luce for providing the illustrations.

\section{REFERENCES}

Alpern M. (1969) Types of movement. In The Eye Vol. 3 (Edited by Davson H.) Chap. 5. pp. 65-174. Academic Press, New York.

Becker W. and Fuchs A. F. (1969) Further properties of the human saccadic system: Eye movements and correction saccades with and without visual fixation points. Vision Res. 9, 1247-1259.

Collewijn H. (1970) The normal range of horizontal eye movements in the rabbit. Exipl Neurol. 28, 132-143.

Dell'Osso L. F. Daroff R. B. and Troost B. T. (1973) Reply to "A comment on the 'glissade'." Vision Res. 13, 883-884.

Easter S. S. (197) Spontaneous eye movements in restrained goldfish. Vision Res. 11, 333-342.

Easter S. S. (1972) Pursuit eye movements in goldfish (Carassius aurafus). 1 ision $R$ ('s. 12, 673-688.

Easter S. S. (1973) A comment on the "glissade". Vision Res. 13, 881-882.

Fuchs A. F. (1967) Saccadic and smooth pursuit eye movements in the monkey. J. Physiol.. Loud. 191. 609-631.

Johnstone J. R. and Mark R. F. (1969) Evidence for efference copy for eye movements in fish. Comp. Biochem. Physiol. 30, 931-939.

Robinson D. A. (1964) The mechanics of human saccadic rye movement. J. Physiol. Lond. 174, 245-264.

Robinson D. A. (1968) Eye movement control in primates. Science, N.Y. 161, 1219-1224.

Weber R. B. and Daroff R. B. (1972) Corrective movements following refixation saccades: type and control system analysis. Vision Res. 12, 467-475.

Westheimer G. (1954) Mechanism of saccadic eye movements. A.M.A. Archs Ophthal. 52, 710-724. 